\title{
Monocyte-to-lymphocyte ratio and platelet-to- lymphocyte ratio in opioid use disorder and marijuana use disorder
}

\author{
Mehmet Hamdi Orum ${ }^{\circledR}$, Mahmut Zabit Kara ${ }^{\circledR}$ \\ ${ }^{1}$ Kahta State Hospital, Department of Psychiatry, Adiyaman - Turkey \\ ${ }^{2}$ Health Sciences University, Antalya Training and Research Hospital, Department of Child and Adolescent Psychiatry, Antalya - Turkey
}

\begin{abstract}
Objective: Various methods are used to determine a person's opioid/marijuana status. However, more specific and sensitive markers are needed that are easily applicable and accessible. Complete blood count $(C B C)$ is a convenient method to be used for this purpose. In this study, we investigated the relationship between $C B C$ values - particularly monocyte-to-lymphocyte ratio (MLR) and platelet-to-lymphocyte ratio (PLR) - and opioid/marijuana use disorder.

Method: We compared CBC values of 56 male patients diagnosed with opioid use disorder (OUD), 56 male patients diagnosed with marijuana use disorder (MUD), and 56 healthy subjects.

Results: The percentage of monocytes was significantly higher in the MUD group compared to the OUD group ( $p=0.010$ ). The monocyte count was significantly different in the MUD group compared to the other two groups $(p=0.018)$. MLR was significantly higher in the MUD group than in the OUD group $(p=0.049)$. PLR was significantly different from the other two groups in the OUD group ( $p=0.038)$. In the MUD group, percentage of monocytes was declining with age $(r=-0.474, p=0.011)$. The area under the receiver operating characteristic curve of the monocyte count value for MUD was 0.670.

Conclusion: Measurements like lymphocyte- and monocyte-related ratios in OUD and MUD can be important in monitoring and detecting the substance and for differentiating between acute and chronic conditions. In order to clarify the subject, further studies with fewer limitations are needed.
\end{abstract}

Keywords: Biomarker, marijuana, monocyte-to-lymphocyte ratio, opioid, platelet-to-lymphocyte ratio

\section{INTRODUCTION}

Substance use disorder (SUD) can result in legal and social problems (1), neuropsychiatric disorders $(2,3)$, infectious diseases, gastrointestinal diseases, intentional and unintentional injuries, cardiovascular diseases, and a number of hematologic changes including thrombocytopenia, thrombocytopenic purpura, toxic granulation of neutrophils, anemia, lymphocytopenia, eosinophilia, and leukocytosis $(1,4,5)$.

Lymphocyte-related ratios are values obtained by the combined calculation of two parameters measured by complete blood count (CBC) (6,7). Platelet-tolymphocyte ratio (PLR) and neutrophil-to-lymphocyte ratio (NLR) are current parameters added to inflammation markers (8-10). Ozkan et al. (11) showed

How to cite this article: Orum MH, Kara MZ. Monocyte-to-lymphocyte ratio and platelet-to-lymphocyte ratio in opioid use disorder and marijuana use disorder. Dusunen Adam The Journal of Psychiatry and Neurological Sciences 2020;33:139-145.

Correspondence: Mehmet Hamdi Orum, Kahta State Hospital, Department of Psychiatry, 02100, Adiyaman - Turkey 
that patients with heroin dependence tended to have a statistically significantly higher NLR compared to healthy controls. According to the study by Cicek et al. (12), the mean NLR and PLR levels of patients with heroin dependence were significantly higher than in control subjects. Oseni et al. (13) stated that the NLR did not differ between marijuana use disorder (MUD) patients and controls. Guzel et al. (14) reported that mean platelet volume (MPV) and percentage of lymphocytes (LYM\%) were significantly lower in synthetic cannabinoid use disorder compared to healthy controls; although there was a statistically significant difference between groups regarding NLR, there was no significant difference for PLR values. Orum et al. (5) reported that PLR, monocyteto-lymphocyte ratio (MLR), and percentage of monocytes (MONO\%) were significantly lower in the opioid use disorder (OUD) group compared to healthy controls. To the best of our knowledge, there is no study comparing the lymphocyte-related ratios between OUD and MUD. In this study, we aimed to examine the MLR, PLR, basophil-to-lymphocyte ratio (BLR), and NLR together with other $\mathrm{CBC}$ values in SUD and investigate the diagnostic value of the lymphocyte-related ratios, especially MLR and PLR, in SUD.

\section{METHOD}

\section{Study Population}

In this retrospective cohort study, we compared the $\mathrm{CBC}$ of 56 male patients diagnosed with OUD and 56 male patients diagnosed with MUD without any psychiatric treatment history who had been admitted to the Alcohol and Drug Addiction Research Treatment and Training Center (AMATEM) outpatient clinic of Kahta State Hospital and diagnosed with OUD (with the method of snorting) and MUD, respectively, with the data of 56 male healthy subjects with a similar age distribution. Parameters assessed were white blood cell (WBC) and red blood cell (RBC) count, hemoglobin (HGB), hematocrit (HTC), mean corpuscular volume (MCV), mean corpuscular hemoglobin $(\mathrm{MCH})$, mean corpuscular hemoglobin concentration $(\mathrm{MCHC})$, red blood cell distribution width (RDW-CV), platelet count (PLT), platelet distribution width (PDW), plateletcrit (PCT) and MPV, neutrophil (NEU), lymphocyte (LYM), NLR, PLR, BLR, and MLR. Local ethics committee approval was obtained, and all study participants provided written informed consent (2019/7-7).

The patient groups consisted of individuals with no substance detected in the urine and without any symptoms of withdrawal or intoxication. According to their preferred type of substance (detected in their urine toxic screening tests up to 1 month previously and confirmed verbally), they were classified as MUD or OUD, respectively. The control group consisted of healthy male volunteers recruited from the hospital staff who did not have a personal or family history of any SUD.

\section{Inclusion Criteria}

Included in the study were patients diagnosed with SUD according to the criteria of the DSM-5 (15) attending the AMATEM outpatient clinic every 2 weeks regularly and whose urine toxic screening test had been negative for the last 4 weeks. According to the toxicological analysis findings, only MUD and OUD were included in the patient group. The intelligence level of all participants was within normal limits.

\section{Exclusion Criteria}

Candidates whose toxicological analysis findings revealed use of substances other than marijuana and opioids were excluded from the patient group. Ten patients with benzodiazepine, 14 patients with stimulants, 7 patients with phencyclidine, 22 patients with sedative-acting antipsychotics, 2 patients with biperiden, 25 patients with ethyl glucuronide, and 18 patients with multiple drug use were thus excluded from the study. Individuals whose information in the patient registry system was incomplete were not included in the study. For this reason, 11 patients were excluded. Patients with comorbid psychiatric diagnosis, hypertension, diabetes mellitus, or severe neurological, immunological, or systemic diseases according to the patient registry system were also excluded. In the control group, 9 people with a substance use history, 12 people who used alcohol, and 6 people whose recorded information was incomplete were therefore excluded from the study. The healthy controls did not have any psychiatric diagnoses, hypertension, diabetes mellitus, or severe neurological, immunological or systemic diseases that might affect the results. Thirteen patients with dull intelligence, 7 patients with borderline intelligence, and 4 patients with mild mental retardation were excluded from the study.

\section{Hematological Analysis}

Venous blood samples of patients and healthy controls were obtained from the antecubital vein between 8 and 10 a.m. after at least 8 hour of fasting. The samples were centrifuged within 30 minutes, and on the same day, centrifugation in the CELL-DYN 3700 SL analyzer 
(Abbott Diagnostics, Chicago, U.S.A.) at the Kahta State Hospital biochemistry laboratory followed. Reference intervals were set as PLT: $142-424\left(10^{3} / \mu \mathrm{L}\right)$, PDW: 0-1000 (fL), PCT: 0-1000 (fL), MPV: 6.8-10.8 (fL), RBC: 4.04-6.13 (10\% $/ \mu \mathrm{L}), \mathrm{MCV}: 80-97$ (fL), MCH: 25-33.5 (pg), RDW-CV: 11.6-15.8 (\%).

\section{Statistical Analysis}

SPSS for Windows version 22 (Armonk, NY: IBM Corp.) was used for all statistical analyses. Numerical data were expressed as mean and standard deviation and categorical data were expressed as frequencies and percentages. Normal distribution was assessed using visual and analytical methods (Kolmogorov-Smirnov/ Shapiro-Wilk test). Student's t-test was used for normal distributions, and one-way ANOVA was used for three independent groups. Mann-Whitney U test and Kruskal Wallis test were used for non-normally distributed data. A post-hoc Tukey honestly significant difference test was used when a significant difference was found between the three independent groups. The relationship between the variables was assessed by Spearman correlation test. Receiver operating characteristic (ROC) curve analysis was used to measure the diagnostic value of the monocyte count (MONO). A value of less than 0.05 was considered statistically significant.

\section{RESULTS}

The mean age was $23.64 \pm 5.30$ years in the OUD group, $23.75 \pm 5.55$ years in the MUD group, and $26.21 \pm 4.80$ years in the control group. The sample groups and the control group consisted of males. There was no significant difference in age between the two groups $(\mathrm{p}=0.121)$. The mean duration of OUD was $4.98 \pm 4.42$ years, the mean duration of MUD 6.42 \pm 5.16 years.

According to the comparison of $\mathrm{CBC}$ values with Student's t-test, MONO\% was significantly higher in the MUD group compared to the OUD group $(\mathrm{p}=0.010)$, but $\mathrm{MONO} \%$ in the MUD group was lower than in the control group, while in the OUD group it was higher than in the control group; however, these differences were not significant. MONO in the MUD group was significantly different from the other two groups ( $p=0.018$ ). MLR was significantly higher in the MUD group compared to the OUD group ( $\mathrm{p}=0.049)$. There were no significant differences between the control group and the other two groups for MLR. PLR was significantly different from the other two groups in the OUD group ( $p=0.038)$. There was no significant difference between groups regarding NLR and BLR
( $\mathrm{p}=0.970$ and $\mathrm{p}=0.473$, respectively). RDW-CV showed a significant difference between all groups $(\mathrm{p}<0.001)$. Other variables showed no significant differences. A comparison of sociodemographic variables and $\mathrm{CBC}$ values of the OUD, MUD, and control groups is given in Table 1.

Spearman's correlation analysis findings between age and $\mathrm{CBC}$ values are also presented in Table 2 separately. In the OUD group, the percentage of neutrophils (NEU\%) was positively correlated with age $(\mathrm{r}=0.507, \mathrm{p}=0.010)$, and LYM\% and MONO\% was declining with age $(\mathrm{r}=-0.472, \mathrm{p}=0.017 ; \mathrm{r}=-0.458$, $\mathrm{p}=0.021$ ). In the MUD group, MONO\% was declining with age $(\mathrm{r}=-0.474, \mathrm{p}=0.011)$. There was no correlation with age in the control group (Table 2).

ROC curve analysis performed to assess the diagnostic value of MONO is shown in Figure 1. The area under the ROC curve of the MONO value for MUD was 0.670 . The optimal cutoff value for MONO was 0.55 , and its sensitivity and specificity for diagnosis of MUD were $64.3 \%$ and $48.1 \%$, respectively.

\section{DISCUSSION}

Our study suggests a correlation between hematological parameters and SUD, especially regarding the immune cells. There was no change in parameters associated

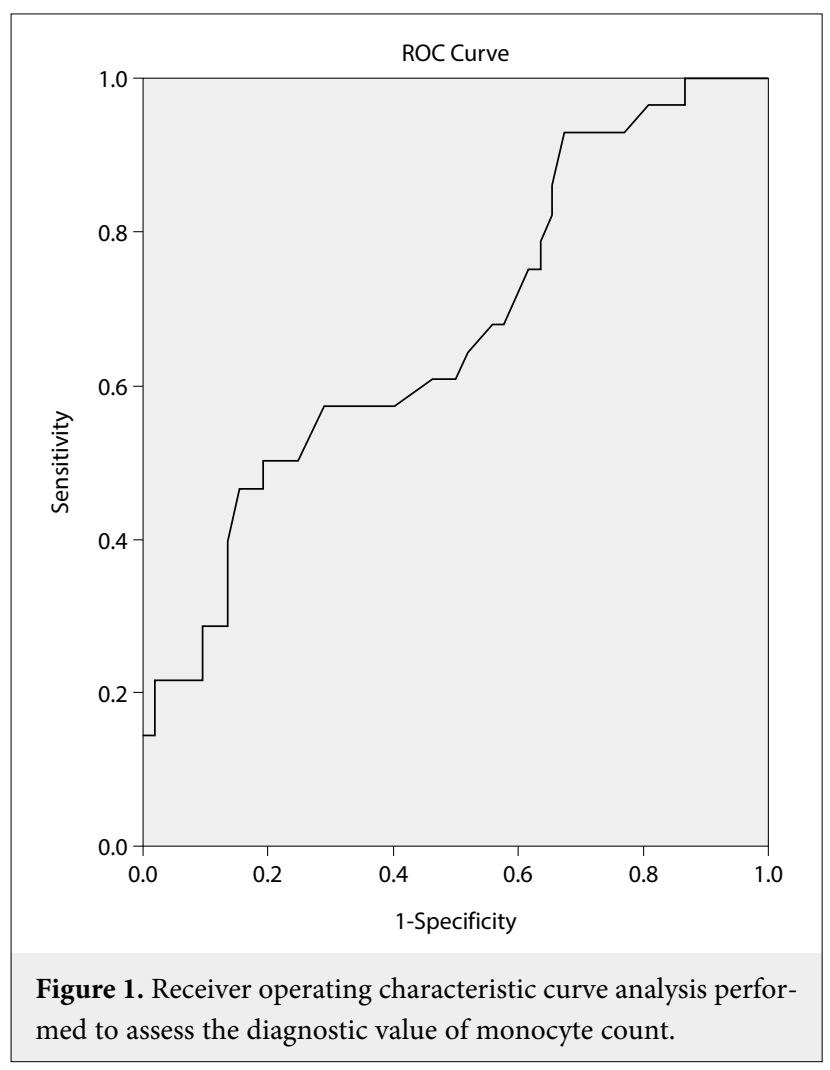


Table 1: Comparison of sociodemographic variables and complete blood count values of male patients with OUD $(n=56)$ and MUD ( $n=56)$, and male control group $(n=56)$

\begin{tabular}{|c|c|c|c|c|c|c|c|c|}
\hline & \multicolumn{2}{|c|}{ OUD $(n=56)$} & \multicolumn{2}{|c|}{ MUD (n=56) } & \multicolumn{2}{|c|}{ Control $(n=56)$} & \multirow[b]{2}{*}{$n^{2}$} & \multirow[b]{2}{*}{ p } \\
\hline & Mean & SD & Mean & SD & Mean & SD & & \\
\hline Age (years) & 23.64 & 5.30 & 23.75 & 5.55 & 26.21 & 4.80 & 0.051 & 0.121 \\
\hline WBC $\left(10^{3} / \mu \mathrm{L}\right)$ & 9.51 & 2.68 & 9.31 & 3.20 & 8.05 & 1.74 & 0.059 & 0.084 \\
\hline $\operatorname{RBC}\left(10^{6} / \mu \mathrm{L}\right)$ & 5.26 & 0.51 & 5.35 & 0.43 & 5.29 & 0.39 & 0.006 & 0.779 \\
\hline HGB (g/dL) & 15.49 & 1.23 & 15.80 & 1.59 & 15.82 & 0.86 & 0.015 & 0.541 \\
\hline HTC (\%) & 46.32 & 3.66 & 47.34 & 4.12 & 46.97 & 2.68 & 0.015 & 0.551 \\
\hline MCV (fL) & 88.32 & 4.63 & 88.49 & 4.82 & 88.89 & 4.23 & 0.003 & 0.891 \\
\hline $\mathrm{MCH}(\mathrm{pg})$ & 29.49 & 1.97 & 29.52 & 1.95 & 29.98 & 2.04 & 0.013 & 0.582 \\
\hline $\mathrm{MCHC}(\mathrm{g} / \mathrm{dL})$ & 33.38 & 1.19 & 33.35 & 1.30 & 33.72 & 1.37 & 0.017 & 0.504 \\
\hline RDW-CV (\%) & 11.69 & 0.63 & 12.17 & 0.92 & 11.19 & 0.98 & 0.183 & $<0.001$ \\
\hline 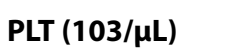 & 233.63 & 55.52 & 249.68 & 68.54 & 245.42 & 49.96 & 0.014 & 0.571 \\
\hline PDW (fL) & 19.28 & 1.38 & 19.34 & 1.65 & 18.54 & 1.53 & 0.055 & 0.099 \\
\hline РСТ (\%) & 0.17 & 0.04 & 0.18 & 0.04 & 0.17 & 0.03 & 0.017 & 0.498 \\
\hline MPV (fL) & 7.81 & 1.83 & 7.91 & 1.91 & 7.52 & 1.14 & 0.010 & 0.665 \\
\hline $\operatorname{NEU}\left(10^{6} / \mu \mathrm{L}\right)$ & 5.85 & 2.71 & 5.79 & 2.76 & 4.71 & 1.70 & 0.012 & 0.154 \\
\hline $\operatorname{LYM}\left(10^{3} / \mu \mathrm{L}\right)$ & 2.77 & 0.62 & 2.48 & 0.62 & 2.50 & 0.78 & 0.037 & 0.214 \\
\hline MONO $\left(10^{3} / \mu \mathrm{L}\right)$ & 0.55 & 0.16 & 0.72 & 0.33 & 0.55 & 0.17 & 0.099 & 0.018 \\
\hline BASO $\left(10^{3} / \mu \mathrm{L}\right)$ & 0.08 & 0.03 & 0.08 & 0.03 & 0.08 & 0.03 & 0.001 & 0.967 \\
\hline $\operatorname{EOS}\left(10^{3} / \mu \mathrm{L}\right)$ & 0.22 & 0.14 & 0.21 & 0.11 & 0.18 & 0.17 & 0.009 & 0.707 \\
\hline NEU\% & 58.33 & 9.85 & 60.14 & 8.89 & 57.82 & 9.42 & 0.012 & 0.629 \\
\hline LYM\% & 31.86 & 8.53 & 28.32 & 7.52 & 33.92 & 8.77 & 0.042 & 0.191 \\
\hline MONO\% & 6.27 & 1.73 & 7.96 & 2.41 & 6.87 & 1.79 & 0.112 & 0.010 \\
\hline BASO $\%$ & 0.97 & 0.31 & 1.00 & 0.31 & 1.05 & 0.33 & 0.009 & 0.697 \\
\hline EOS\% & 2.53 & 1.59 & 2.53 & 1.34 & 2.34 & 2.03 & 0.003 & 0.897 \\
\hline NLR & 2.30 & 1.61 & 2.38 & 1.09 & 2.43 & 2.74 & 0.001 & 0.970 \\
\hline PLR & 87.50 & 25.46 & 103.49 & 30.78 & 107.90 & 39.80 & 0.088 & 0.038 \\
\hline MLR & 0.20 & 0.07 & 0.29 & 0.12 & 0.25 & 0.18 & 0.073 & 0.049 \\
\hline BLR & 0.03 & 0.01 & 0.03 & 0.01 & 0.04 & 0.05 & 0.023 & 0.403 \\
\hline
\end{tabular}

One-Way ANOVA and Post-Hoc Tukey were used. Degrees of freedom=167

$\eta^{2}$ : Effect Size, OUD: Opioid use disorder, MUD: Marijuana use disorder, WBC: White blood cell, RBC: Red blood cell, HGB: Hemoglobin, HTC: Hematocrit, MCV: Mean corpuscular volume, MCH: Mean corpuscular hemoglobin, MCHC: Mean corpuscular hemoglobin concentration, RDW-CV: Red blood cell distribution width-coefficent of variation, PLT: Platelets, PDW: Platelet distribution width, PCT: Plateletcrit, MPV: Mean platelet volume, NEU: Neutrophils, LYM: Lymphocytes, MONO: Monocytes, BASO: Basophils, EOS: Eosinophils, NEU\%: Percentage of neutrophils, LYM\% Percentage of lymphocytes, MONO\%: Percentage of monocytes, BASO\%: Percentage of basophils, EOS\%: Percentage of eosinophils, NLR: Neutrophil to lymphocyte ratio, PLR: Platelet to lymphocyte ratio,

MLR: Monocyte-to-lymphocyte ratio, BLR: Basophil-to-lymphocyte ratio

with red blood cells except RDW-CV. The duration of SUD was greater in the MUD group. According to the literature, the age at starting marijuana use is lower than the age at starting to use opioids. This result is compatible with the literature. There was a significant relationship between PLR and OUD. Our results are similar to some statements in the literature, but incompatible with some others. Rasheed and Iqtidar (16) investigated the hematologic parameters of 100 heroin dependents and suggested a statistically significant decrease in HGB, HTC, RBC, and PLT in heroin addicts compared to control subjects. Verde Mendez et al. (17) demonstrated that for both sexes, the levels of HTC and HGB were similar in the control groups and in opiate addicts. Savov et al. (18) established a heroin macrocytosis in chronic heroin abusers. Again, there was no change in parameters associated with platelets. Guzel et al. (14) designed a study finding WBC, MCH, RDW-CV, MCV, MPV, MONO, and NEU parameters to be significantly higher in the synthetic cannabinoid group compared to the control group. 
Table 2: The Correlation between age and complete blood count parameters of the groups

\begin{tabular}{|c|c|c|c|c|c|c|c|c|}
\hline \multirow[b]{2}{*}{ Parameters } & \multicolumn{2}{|c|}{ OUD } & \multicolumn{3}{|c|}{ MUD } & \multicolumn{3}{|c|}{ Control } \\
\hline & $\mathbf{r}$ & $\mathbf{p}$ & Parameters & $\mathbf{r}$ & $\mathbf{p}$ & Parameters & $\mathbf{r}$ & $\mathbf{p}$ \\
\hline NEU\% & 0.507 & 0.010 & NEU\% & 0.111 & 0.575 & NEU\% & -0.246 & 0.217 \\
\hline LYM\% & -0.472 & 0.017 & LYM\% & -0.023 & 0.909 & LYM\% & 0.215 & 0.281 \\
\hline MONO\% & -0.458 & 0.021 & MONO\% & -0.474 & 0.011 & MONO\% & -0.013 & 0.949 \\
\hline BASO $\%$ & -0.248 & 0.233 & BASO\% & 0.198 & 0.313 & BASO\% & 0.091 & 0.653 \\
\hline EOS\% & -0.069 & 0.742 & EOS\% & 0.200 & 0.307 & EOS\% & 0.182 & 0.364 \\
\hline MONO & 0.047 & 0.824 & MONO & -0.349 & 0.069 & MONO & -0.023 & 0.910 \\
\hline BASO & 0.206 & 0.324 & BASO & 0.102 & 0.604 & BASO & -0.039 & 0.845 \\
\hline EOS & 0.202 & 0.333 & EOS & 0.261 & 0.179 & EOS & 0.172 & 0.391 \\
\hline BLR & 0.210 & 0.313 & BLR & 0.141 & 0.473 & BLR & -0.084 & 0.677 \\
\hline MLR & 0.101 & 0.631 & MLR & -0.331 & 0.086 & MLR & -0.155 & 0.440 \\
\hline
\end{tabular}

$\mathrm{p}<0.05$, Spearmen Correlation Analysis was used.

OUD: Opioid use disorder, MUD: Marijuana use disorder, NEU\%: Percentage of neutrophils, LYM\%: Percentage of lymphocytes, MONO\%: Percentage of monocytes, BASO\%: Percentage of basophils, EOS\%: Percentage of eosinophils, MONO: Monocyte count, BASO: Basophil count, EOS: Eosinophil count,

BLR: Basophil-to-lymphocyte ratio, MLR: Monocyte-to-lymphocyte ratio

On the other hand, our results suggest that the use of opioids and marijuana causes a change in the immune response and these results are consistent with the literature. Opioids have been shown to inhibit immune parameters such as natural killer (NK) activity, lymphocyte proliferation, and nitric oxide production. As is well known, opioids may effect neural modulation of immunity through both neuroendocrine and autonomic efferent pathways $(19,20)$. Banerjee and Sarkar's study (21) indicates that the MONO was significantly lower in heroin smokers than in control persons. Perez-Castrillon et al. (22) suggest that opioids play an important role in the depression of monocyte chemotaxis observed in intravenous drug abusers. Novick et al. (23) reported that NK activity was reduced significantly in parenteral heroin abusers compared with methadone maintenance patients and apparently healthy individuals. $\Delta(9)$-tetrahydrocannabinol (THC), the major product of marijuana, exhibits immunosuppressive activity. Another product of marijuana, cannabidiol (CBD), while not psychotropic, also modulates immune function $(24,25)$. THC inhibits the capability of macrophage-like cells residing in the central nervous system, such as microglia, to migrate toward nodes of microbial invasion. It has been reported that $\mathrm{THC}$ and $\mathrm{CBD}$ have developmental and long-term effects on the immune system, indicating that exposure to these substances early in life might alter the fundamental neuroimmune response to certain microbial agents in adults (24).

Endocannabinoid receptors, notably the cannabinoid receptor type 2 , play a role in the development and modulation of immune and hematologic cells. Studies performed with chronic marijuana consumers demonstrate the capacity of this substance to reduce the number of $T$ and $B$ lymphocytes and increase the number of eosinophils $(13,24,26)$. According to our study, the use of marijuana affects MONO\% more than opioid use. Again, MONO is associated with marijuana use but not with opioid use. MONO\% correlated with age in the MUD group. These results and the ROC curve analysis suggest that the use of marijuana and monocyte-related parameters are related. In our study, monocyte-associated biomarkers were found to be more relevant to marijuana use than opioid use. Promonocytes and their precursors in the bone marrow, monocytes in the peripheral blood, and the macrophages in the tissues are all part of the mononuclear phagocyte system (27). Macrophages are of major importance both in innate immunity to infections, producing acute-phase cytokines as well as phagocytosing and killing microbes, and in acquired immunity, where they present antigen and release cytokines (28). Studies of lung alveolar macrophages showed marijuana smoking to have little effect on phagocytic capacity, while it caused some metabolic and morphological changes in the cells $(24,27)$.

Self-report with instruments such as Addiction Severity Index, Addiction Profile Index, and Drug Abuse Screening Test is inexpensive and relatively accurate, but it has limitations particularly due to participant's denial or recall bias $(29,30)$. For this reason, laboratory evidence of OUD and MUD is needed. Measures related to OUD and MUD are mostly found in the field of forensic and emergency medicine. 
Measurements in OUD and MUD are important for substance monitoring and detection. It is also important to monitor the treatment response and determine the level of compliance (31). Clinicians and patients can avail themselves of biomarkers to gather information about the current status or future risk of disease (32). Using hematologic parameters is an easy and inexpensive method in cases where we cannot get unequivocal information from patients. For instance, an important research area is associated with hematologic markers of alcoholism. For alcohol users, the markers known as state markers are divided into two groups: relapse markers and screening markers. Relapse markers are also sensitive to acute alcohol consumption. Screening markers may be useful for early identification before alcohol use disorder emerges. Carbohydrate-deficient transferrin, RBC, and MCV are the hematological state markers used for this purpose $(33,34)$. Increasing biomarker work related to heroin, as in alcohol, will facilitate the planning of follow-up and treatment of these patients, increase utility in legal cases, and can be used to differentiate between chronic and acute conditions. In our study, there were changes in MONO, MONO\%, MLR, and PLR. CBC values and calculations made based on both of these values are intended to be used as OUD and MUD biomarkers.

As a conclusion, the known effect of opioid and marijuana on lymphocytes, neutrophils, platelets led us to investigate the effect of OUD on PLR, MLR, BLR, and NLR. There is a relationship between OUD and MUD and hematological parameters including $\mathrm{MONO} \%$ and $\mathrm{RDW}-\mathrm{CV}$. Retrospectively, our $\mathrm{CBC}$ results showed that OUD and MUD were correlated with PLR and MLR but not with BLR and NLR. This study provides the opportunity to determine NLR, BLR, PLR and MLR values of OUD and MUD patients in one study.

Our study has several limitations, most importantly due to its retrospective design; therefore, prospective studies are needed. Furthermore, evaluating data from larger samples according to gender and age groups appears to be necessary. Studies with larger samples will reveal more clearly the relationship between opioids, marijuana, and MLR and PLR. Long-term studies are needed to follow the course of disease after detoxification and remission periods. The frequency and duration of substance use are further important points that may affect blood parameters. Studies are needed to clarify these points. The possible effect of smoking on the results is unknown. Lifestyle and nutritional characteristics of the individual are additional confounding factors. Further studies are needed to confirm the present results and explain the underlying mechanisms.

\begin{tabular}{|c|c|c|}
\hline \multicolumn{2}{|c|}{ Contribution Categories } & \multirow{2}{*}{$\begin{array}{l}\text { Author Initials } \\
\text { M.H.O., M.Z.K. }\end{array}$} \\
\hline \multirow{3}{*}{ Category 1} & Concept/Design & \\
\hline & Data acquisition & M.H.O., M.Z.K. \\
\hline & Data analysis/Interpretation & M.H.O., M.Z.K. \\
\hline \multirow{2}{*}{ Category 2} & Drafting manuscript & M.H.O., M.Z.K. \\
\hline & Critical revision of manuscript & M.H.O., M.Z.K. \\
\hline Category 3 & Final approval and accountability & M.H.O., M.Z.K. \\
\hline \multirow{2}{*}{ Other } & Technical or material support & M.H.O., M.Z.K. \\
\hline & Supervision & M.H.O., M.Z.K. \\
\hline
\end{tabular}

Ethics Committee Approval: Ethical approval was obtained from Adiyaman University (Protocol number: 2019/7-7).

Informed Consent: Written informed consent was obtained from the patient.

Peer-review: Externally peer-reviewed.

Conflict of Interest: The authors declared that there were no conflicts of interest regarding the publication of this paper.

Financial Disclosure: The authors declared that this study has received no financial support.

\section{REFERENCES}

1. United Nations Office on Drugs and Crime. World Drug Report 2016. United Nations publication Sales No. E.16.XI.7.

2. Egilmez OB, Orum MH, Dumlupinar E, Egeli A. Retrospective evaluation of the applications within the scope of probation implementation: The data of 2018. Journal of Dependence 2019; 20:80-87. (Turkish)

3. Orum MH, Kustepe A, Kara MZ, Dumlupinar E, Egilmez OB, Ozen ME, Kalenderoglu A. Addiction profiles of patients with substance dependency living in Adiyaman province. Med Sci (Turkey) 2018; 7:369-372.

4. Dangana A, Nasir IA, Medugu JT, Emeribe AU. Haematological changes associated with illicit drug abuse in a city of Northern Nigeria. Int J Hematol Res 2016; 2:160-163.

5. Orum MH, Kara MZ, Egilmez OB, Kalenderoglu A. Complete blood count alterations due to the opioid use: what about the lymphocyte-related ratios, especially in monocyte to lymphocyte ratio and platelet to lymphocyte ratio? J Immunoassay Immunochem 2018; 39:365-376.

6. Kara MZ, Orum MH, Sekmen E. Is there a relationship between basophil, platelet-related parameters and developmental stuttering? Anadolu Psikiyatri Derg 2020; 21:187-194.

7. Orum MH, Kara MZ. Platelet to lymphocyte ratio (PLR) in alcohol use disorder. J Immunoassay Immunochem 2020; 41:184-194.

8. Orum MH, Kara MZ, Egilmez OB. Relationship between immune cells and alcohol dependents and controls: what about the lymphocyte-related ratios? J Immunoassay Immunochem 2018; 39:348-350. 
9. Kara MZ, Orum MH, Egilmez OB. Relationship between immune cells and violent/nonviolent suicide attempts and controls: What about the lymphocyte-related ratios and neutrophil-related parameters? Kaohsiung J Med Sci 2019; 35:315-316.

10. Orum MH, Kara MZ, Egilmez OB. Mean platelet volume and neutrophil to lymphocyte ratio as parameters to indicate the severity of suicide attempt. J Immunoassay Immunochem 2018; 39:647-659.

11. Ozkan N, Sonmez MB, Tas Durmus P, Gorgulu Y, Kose Cinar R, Vardar ME. Elevated neutrophil lymphocyte ratio in patients with substance use disorders. Eur Psychiatry 2017; 41(Suppl):S872-S873.

12. Cicek E, Demirel B, Cicek IE, Kirac AS, Eren I. Increased neutrophil-lymphocyte and platelet-lymphocyte ratios in male heroin addicts: A prospective controlled study. Clin Psychopharmacol Neurosci 2018; 16:190-196.

13. Oseni BS, Togun VA, Taiwo OF. Effect of marijuana smoking on some hematological parameters of smokers. World Journal of Medical Sciences 2006; 1:82-85.

14. Guzel D, Yazici AB, Yazici E, Erol A. Alterations of the hematologic cells in synthetic cannabinoid users. J Clin Lab Anal 2017; 31:e22131.

15. American Psychiatric Association. Diagnostic and statistical manual of mental disorders. Fifth ed,. Washington, DC: American Psychiatric Association, 2013.

16. Rasheed A, Iqtidar A. Hematological profile, serum electrolytes and iron level investigations in heroin addicts. Acta Pharmaceutica Turcica 1997; 39:59-63.

17. Verde Méndez CM, Díaz-Flores JF, Sañudo RI, Rodríguez Rodríguez EM, Díaz Romero C. Haematologic parameters in opiate addicts. Nutr Hosp 2003; 18:358-365. (Spanish).

18. Savov Y, Antonova N, Zvetkova E, Gluhcheva Y, Ivanov I, Sainova I. Whole blood viscosity and erythrocyte hematometric indices in chronic heroin addicts. Clin Hemorheol Microcirc 2006; 35:129-133.

19. Fecho K, Nelson CJ, Lysle DT. Phenotypic and functional assessments of immune status in the rat spleen following acute heroin treatment. Immunopharmacology 2000; 46:193-207.

20. Saurer TB, Ijames SG, Lysle DT. Evidence for the nucleus accumbens as a neural substrate of heroin-induced immune alterations. J Pharmacol Exp Ther 2009; 329: 1040-1047.
21. Banerjee D, Sarkar NK. A study of some blood parameters in chronic heroin smokers. Med Sci Res 1994; 22:379-380.

22. Pérez-Castrillón JL, Pérez-Arellano JL, García-Palomo JD, Jiménez-López A, De Castro S. Opioids depress in vitro human monocyte chemotaxis. Immunopharmacology 1992; 23:57-61.

23. Novick DM, Ochshorn M, Ghali V, Croxson TS, Mercer WD, Chiorazzi N, et al. Natural killer cell activity and lymphocyte subsets in parenteral heroin abusers and long-term methadone maintenance patients. J Pharmacol Exp Ther 1989; 250:606-610.

24. Cabral GA, Jamerson M. Marijuana use and brain immune mechanisms. Int Rev Neurobiol 2014; 118:199-230.

25. Cabral GA, Rogers TJ, Lichtman AH. Turning over a new leaf: Cannabinoid and endocannabinoid modulation of immune function. J Neuroimmune Pharmacol 2015; 10:193-203.

26. Coppola M, Mondola R. Cannabis consumption systemic adverse effects. Int J High Risk Behav Addict 2014; 3:e18627.

27. van Furth R, Cohn ZA, Hirsch JG, Humphrey JH, Spector WG, Langevoort HL. The mononuclear phagocyte system: a new classification of macrophages, monocytes, and their precursor cells. Bull World Health Organ 1972; 46:845-852.

28. Klein TW, Newton CA, Friedman H. Cannabinoids and the immune system. Pain Res Manag 2001; 6:95-101.

29. Evren C, Ogel K, Evren B, Bozkurt M. Psychometric properties of the Turkish versions of the Drug Use Disorders Identification Test (DUDIT) and the Drug Abuse Screening Test (DAST-10) in the prison setting. J Psychoactive Drugs 2014; 46:140-146.

30. McLellan AT, Kushner H, Metzger D, Peters R, Smith I, Grissom $\mathrm{G}$, et al. The Fifth Edition of the Addiction Severity Index. J Subst Abuse Treat 1992; 9:199-213.

31. Kintz P, Jamey C, Cirimele V, Brenneisen R, Ludes B. Evaluation of acetylcodeine as a specific marker of illicit heroin in human hair. J Anal Toxicol 1998; 22:425-429.

32. Niemelä O. Biomarker-Based Approaches for Assessing Alcohol Use Disorders. Int J Environ Res Public Health 2016; 13:166.

33. Esel E. Biological trait markers of alcohol dependence. Turk Psikiyatri Derg 2003; 14:60-71. (Turkish).

34. Youn K, Kim JS, Kim SS, Yoon SJ, Woo DJ. Carbohydratedeficient transferrin as a biomarker for screening at-risk drinking in elderly men. Korean J Fam Med 2017; 38:291-295. 